\title{
ON THE CONSISTENCY OF SOBOL INDICES WITH RESPECT TO STOCHASTIC ORDERING OF MODEL PARAMETERS
}

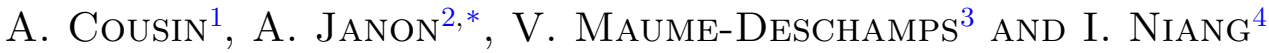

\begin{abstract}
In the past decade, Sobol's variance decomposition has been used as a tool to assess how the output of a model is affected by the uncertainty on its input parameters. We show some links between global sensitivity analysis and stochastic ordering theory. More specifically, we study the influence of inputs' distributions on Sobol indices in relation with stochastic orders. This gives an argument in favor of using Sobol's indices in uncertainty quantification, as one indicator among others.
\end{abstract}

Mathematics Subject Classification. 62P99.

Received January 22, 2017. Accepted January 3, 2018.

\section{INTRODUCTION}

Many models encountered in applied sciences involve input parameters which are often not precisely known. Sobol indices are used so as to assess the sensitivity of a model output as a function of its input parameters. In other words, they quantify the impact of inputs' uncertainty on an output. Sobol indices are widely used for example in hydrology (see $[19,27])$ and more generally for environmental and industrial applications (see, e.g., $[11,28])$.

Recently $[4,18,21]$, this global sensitivity analysis has been used as a risk management tool, amongst other indicators [3]. These indices are set in a framework which requires, as a postulate, the definition of a probability distribution for the input parameters of the model, so as to quantify, as a prior, the uncertainty on the inputs. As noted by [20], Sobol indices (and, more generally, any sensitivity measure) crucially depend on the chosen probability distribution on the inputs. At the same time, practitioners often experience difficulties to choose this inputs' probability distribution.

Hence, it appears that analyzing the influence of the inputs' distribution on the Sobol indices of an output is an important question. To our knowledge, while such empirical studies (on a particular output, with particular inputs) have been conducted (see [20] e.g.), and perturbation of inputs' distribution have been considered (see $[1,15])$, no theoretical analysis comparable to ours has been performed yet.

This paper aims at beginning this analysis by proving that Sobol indices behave consistently with respect to some stochastic orders. This question arises naturally: under some reasonable conditions on the output,

Keywords and phrases: Sensitivity analysis, Sobol indices, stochastic orders.

${ }^{1}$ Institut de Recherche en Mathématique Avancée, Université de Strasbourg, France.

${ }^{2}$ Université Paris Sud (Paris Saclay), Laboratoire de Mathématiques d'Orsay, CNRS UMR 8628, France.

3 Université de Lyon, Université Lyon 1, Institut Camille Jordan ICJ UMR 5208 CNRS, France.

${ }^{4}$ Université de Lyon, Université Lyon 1, Laboratoire SAF EA 2429, France.

* Corresponding author: alexandre.janon@math.u-psud.fr 
uncertainty quantifiers should increase if the uncertainty on the input increases in some way. Thus, our problematic is to find for which kind of stochastic orders and under which sufficient conditions on the output function the Sobol indices behave consistently.

Roughly speaking, given two random variables $X$ and $Y$, the $X$-Sobol index on $Y$ is given by

$$
S_{X}=\frac{\operatorname{Var}(\mathbb{E}(Y \mid X))}{\operatorname{Var}(Y)} .
$$

It is a statistical indicator of the relative impact of $X$ on the variability of $Y$. If we study the impact of several independent variables $X_{1}, \ldots, X_{k}$ on $Y$, then the Sobol indices may be used to provide a hierarchization of the $X_{i}$ 's with respect to their impact on $Y$. It is an interesting alternative to regression coefficient, which may be difficult to interpret if the relationship between $Y$ and the $X_{i}$ 's is far from linear.

In our context, the uncertainty on the input parameters is described by a random vector $X=\left(X_{1}, \ldots, X_{k}\right)$ of $\mathbb{R}^{k}$, with the $X_{i}$ 's being independent. This classical hypothesis ensures that the Sobol indices are well-defined. Even if recent works deal with dependent input variables (see e.g. [16]), we restrict ourselves to the classical theory of Sobol indices. We are interested in the Sobol indices of an output function $f$. One of our main result is that, under some convexity or monotonicity assumptions on $f$, Sobol indices have a behavior which is compatible with respect to the excess wealth order or the dispersive order (see Thms. 4.1 and 4.3). This fact confirms that Sobol indices can be used to quantify some uncertainty, even if, depending on the purpose, moment-independent approaches might be preferred to variance decomposition (see [2]).

The paper is organized as follows. In Section 2, we recall the definition of Sobol indices. In Section 3, we provide one example showing that a change of the laws of the inputs has an important impact on Sobol indices. It motivates the theoretical study on the relationship between stochastic orders and Sobol indices. Definitions and elementary properties of stochastic orders are recalled in Appendix A. In Section 4, we state our main results about the consistency of Sobol indices with respect to the dispersive order. In Section 5 we give illustration of our results. Section 6 is devoted to multivariate extensions of the results presented in Section 4. In Section 7, we give some concluding remarks. The main mathematical proofs are postponed to Appendix B.

\section{Definition of Sobol indices}

Understanding the effects of uncertainties in parameter values on a model behaviour is a crucial issue in applied sciences. Sobol indices can be used as a tool to identify the key input parameters that drives the uncertainty on the model output. Let us recall the definitions of Sobol indices. We refer to [6, 25] or [12] for more details on this subject. We consider one output $Y=f\left(X_{1}, \ldots, X_{k}\right)$ of a model given as a deterministic function $f$. The input parameters are uncertain and we assume that they are given as independent random variables $X_{1}, \ldots, X_{k}$.

To simplify notation, we denote by $X^{-i}$ the random vector $\left(X_{1}, \ldots, X_{i-1}, X_{i+1}, \ldots, X_{k}\right)$, for any $i \in\{1, \ldots k\}$. In addition, for any $\alpha \subset\{1, \ldots k\}$ and $X_{\alpha}$ refers to the random vector $\left(X_{i}, i \in \alpha\right), X^{-\alpha}$ refers to the random vector $\left(X_{i}, i \notin \alpha\right)$. Moreover, for any $\alpha \subset\{1, \ldots, k\}$, we denote by $\mu_{X_{\alpha}}$ the law of the random vector $X_{\alpha}$ and by $|\alpha|$ the number of elements in the set $\alpha$.

The function $f$ can be expressed as its Hoeffding decomposition [26]:

$$
f\left(X_{1}, \ldots, X_{k}\right)=\sum_{\alpha \subset\{1, \ldots, k\}} f_{\alpha}\left(X_{\alpha}\right)
$$

with

(1) $f_{\varnothing}=\mathbb{E}(f(X))$,

(2) $\int f_{\alpha} \mathrm{d} \mu_{X_{i}}=0$ if $i \in \alpha$, 
(3) $\int f_{\alpha} \cdot f_{\beta} \mathrm{d} \mu_{X}=0$ if $\alpha \neq \beta$.

The functions $f_{\alpha}$ are defined inductively:

$$
\begin{gathered}
f_{\varnothing}=\mathbb{E}(f(X)), \\
f_{i}\left(X_{i}\right)=\mathbb{E}\left(f(X) \mid X_{i}\right)-f_{\varnothing}=\int f \mathrm{~d} \mu_{X^{-i}}-f_{\varnothing},
\end{gathered}
$$

for $i \in\{1, \ldots, k\}$. Finally, let $\alpha \subset\{1, \ldots, k\}$; then,

$$
f_{\alpha}\left(X_{\alpha}\right)=\int f \mathrm{~d} \mu_{X^{-\alpha}}-\sum_{\beta \subsetneq \alpha} f_{\beta}\left(X_{\beta}\right) .
$$

With these notations, we have that:

$$
\operatorname{Var}(Y)=\operatorname{Var}(f(X))=\sum_{\alpha \subset\{1, \ldots, k\}} \operatorname{Var}\left(f_{\alpha}\left(X_{\alpha}\right)\right)=\sum_{\alpha \subset\{1, \ldots, k\}} \mathbb{E}\left(f_{\alpha}\left(X_{\alpha}\right)^{2}\right) .
$$

The impact of $X_{i}$ on $Y=f(X)$ may be measured by the Sobol index defined as

$$
S_{i}=\frac{\operatorname{Var}\left(\mathbb{E}\left(f(X) \mid X_{i}\right)\right)}{\operatorname{Var}(Y)}=\frac{\mathbb{E}\left(f_{i}\left(X_{i}\right)^{2}\right)}{\operatorname{Var}(Y)},
$$

for $i \in\{1, \ldots k\}$. There are also interactions between the variables $X_{1}, \ldots, X_{k}$, which are encoded into the functions $f_{\alpha}$, with $|\alpha| \geq 2$. The total Sobol indices take into account the impact of the interactions and are defined as

$$
S_{T_{i}}=\frac{\sum_{i \in \alpha \subset\{1, \ldots, k\}} \operatorname{Var}\left(f_{\alpha}\left(X_{\alpha}\right)\right)}{\operatorname{Var}(Y)}=\frac{\sum_{i \in \alpha \subset\{1, \ldots, k\}} \mathbb{E}\left(f_{\alpha}\left(X_{\alpha}\right)^{2}\right)}{\operatorname{Var}(Y)},
$$

for $i \in\{1, \ldots k\}$.

\section{A motivating example}

In this section, we provide a motivating example on a classical financial risk model. Financial models are associated with a set of parameters. In a context where these parameters are not known with certainty (due to estimation error for instance), the global sensitivity analysis is useful to assess which (uncertain) input parameters mostly contribute to the uncertainty of model output, that is, which parameters have to be estimated with care. In most of our examples, we will consider truncated distributions. The use of truncated distributions is motivated by the fact that distributions of financial parameters generally have bounded supports.

Definition 3.1. Let $(a, b) \in \mathbb{R}^{2}$ and let $X$ be a random variable with density function $h$ and cumulative distribution $F$. The truncated distribution of $X$ on the interval $[a, b]$ is the conditional distribution of $X$ given that $a<X \leq b$. Its density function is then given by

$$
h(x \mid a<X \leq b)=\frac{h(x) \mathbf{1}_{a<x \leq b}}{F(b)-F(a)} .
$$


TABLE 1. Total Sobol indices of VaR (3.2) when $\alpha=0.9$. All digits are significant with a $95 \%$ probability.

\begin{tabular}{llllllll}
\hline$\mu^{*}$ & $\sigma^{*}$ & $S_{T_{\mu}}^{*}$ & $S_{T_{\sigma}}^{*}$ & $\mu$ & $\sigma$ & $S_{T_{\mu}}$ & $S_{T_{\sigma}}$ \\
\hline $\mathcal{U}[0,1]$ & $\mathcal{U}[0,1]$ & 0.41 & 0.64 & $\mathcal{U}[0,1]$ & $\mathcal{U}[0,2]$ & 0.20 & 0.87 \\
$\mathcal{U}[0,2]$ & $\mathcal{U}[0,1]$ & 0.73 & 0.36 & $\mathcal{U}[0,2]$ & $\mathcal{N}_{T}(0.5,2)$ & 0.48 & 0.69 \\
$\mathcal{U}[0,1]$ & $\mathcal{E}_{T}(5)$ & 0.53 & 0.52 & $\mathcal{U}[0,1]$ & $\mathcal{E}_{T}(1)$ & 0.4 & 0.66 \\
$\mathcal{U}[0,1]$ & $\mathcal{U}[0,1]$ & 0.40 & 0.65 & $\mathcal{N}_{T}(0.5,2)$ & $\mathcal{U}[0,1]$ & 0.73 & 0.35 \\
\hline
\end{tabular}

In what follows, we denote by $\mathcal{N}_{T}$ and $\mathcal{E}_{T}$ a truncated normal and a truncated exponential laws respectively. In risk management, the Value-at-risk ( $\mathrm{VaR}$ ) is a widely used risk measure of the risk of losses associated with portfolio of financial assets (such as stocks, bonds, etc.). From a mathematical point of view, if $L$ denotes the loss associated with a portfolio of assets, then $\operatorname{VaR}_{\alpha}(L)$ is defined as the $\alpha$-quantile level of this loss, i.e.,

$$
\operatorname{VaR}_{\alpha}(L):=\inf \left\{x \in \mathbb{R}: F_{L}(x) \geq \alpha\right\}
$$

where $F_{L}$ denotes the cumulative distribution function of $L$.

Let us consider a portfolio loss of the form $L=S_{T}-K$ where $K$ is positive and where $S_{T}$ stands for the value at time $T$ of a portfolio of financial assets. This corresponds to the loss at time $T$ of a short position on this portfolio when the latter has been sold at time 0 for the price $S_{0}=K \exp (-r T)$ where $r$ is a constant risk-free interest-rate. We assume that $\left(S_{t}\right)_{t \geq 0}$ follows a geometric brownian motion so that its value at time $T$ can be expressed as

$$
S_{T}=S_{0} \exp \left(\mu T+\sigma W_{T}\right)
$$

where $W_{T}$ is the value at time $T$ of a standard Brownian motion, $\mu$ (resp. $\sigma$ ) is a positive drift (resp. volatility) parameter. Therefore, the $\alpha$-Value-at-Risk associated with the loss $L$ at time $T$ is given by

$$
\operatorname{VaR}_{\alpha}(L)=S_{0} \exp \left(\mu T+\sigma \sqrt{T} \phi^{-1}(\alpha)\right)-K,
$$

where $S_{0} \in \mathbb{R}_{+}^{*}$ and where $\phi^{-1}$ is the normal inverse cumulative distribution function of a standard Gaussian random variable. Note that, as soon as $\alpha \geq 0.5$, the VaR expression (3.2) can be seen as a product of log-convex non-decreasing functions with respect to $\mu$ and $\sigma$.

Our interest is to quantify the sensitivity of the uncertain parameters $\mu$ and $\sigma$ on the Value-at-Risk $\left(\operatorname{VaR}_{\alpha}(L)\right)$ by evaluating the total Sobol indices $S_{T_{\mu}}$ and $S_{T_{\sigma}}$ as defined by (2.4). We then analyze the impact of a change in the input parameters law on the total Sobol indices $S_{T_{\mu}}$ and $S_{T_{\sigma}}$. In the numerical illustrations, we consider a VaR associated with a risk level $\alpha=0.9$ computed on a portfolio loss with the following characteristics:

$$
T=1, S_{0}=100, K=100 .
$$

Table 1 compares the total Sobol indices associated with the input parameters $\mu$ and $\sigma$ under different assumptions on their probability distribution. Each line of the table corresponds to a situation where the distribution of only one parameter has been modified. We notice that the distributions of $\mu$ and $\sigma$ have greater variance, respectively, than the distributions of $\mu^{*}$ and $\sigma^{*}$. As can be seen, changing the laws of model parameters $\mu$ and $\sigma$ has a significant impact on the values of total Sobol indices $S_{T_{\mu}}$ and $S_{T_{\sigma}}$. In addition, these empirical illustrations seem to indicate that the more "risky" or uncertain the parameter, the higher the corresponding total Sobol index. This behavior will be investigated theoretically using the theory of stochastic ordering. 


\section{Relationships Between Stochastic orders And Sobol indices}

In this section, we explore how an increase of riskness (in the sense of stochastic ordering) in the input parameters may have an impact on the model output. In this respect, we consider two particular stochastic orders, that is, the excess wealth order (denoted by ew) and the dispersive order (denoted by disp), whose definitions and main properties are recalled in Appendix A.

\subsection{Relationship with stochastic orders when there is no interaction}

In this section, we assume that there is no interaction between the $X_{i}$ 's, that is, $f(X)$ can be expressed in the following additive form:

$$
f\left(X_{1}, \ldots, X_{k}\right)=\sum_{j=1}^{k} g_{j}\left(X_{j}\right)+K,
$$

where $g_{1}, \ldots, g_{k}$ are real-valued functions and $K \in \mathbb{R}$. It is straightforward to prove that, in that case, decomposition (2.1) reduces to

$$
f(X)=\sum_{i=1}^{k} f_{i}\left(X_{i}\right)+f_{\varnothing},
$$

so that, for any $i=1, \ldots, k$, the "individual" Sobol index defined by (2.3) coincides with the total Sobol index defined by (2.4).

Let $X_{i}^{*}$ denote another variable that will be compared to $X_{i}$. We will assume $X_{i}^{*} \leq$ ew $X_{i}$ and study the impact of replacing $X_{i}$ by $X_{i}^{*}$ on Sobol indices. We assume that $X_{i}^{*}$ is independent of $X^{-i}$ and we denote by $X^{*}=\left(X_{1}, \ldots, X_{i-1}, X_{i}^{*}, X_{i+1}, \ldots, X_{k}\right)$ the vector $X$ where the $i$ th component has been replaced by $X_{i}^{*}$ and by

$$
S_{i}^{*}=\frac{\left.\operatorname{Var}\left(\mathbb{E}\left(f\left(X^{*}\right) \mid X_{i}^{*}\right)\right)\right)}{\operatorname{Var}\left(f\left(X^{*}\right)\right)}
$$

the $i$ th Sobol index associated with $f\left(X^{*}\right)$. Hence, $f\left(X^{*}\right)$ is the output where distribution of the $i$ th input distribution has been modified, and the others left unchanged.

As we consider the excess wealth order, we assume that $X_{i}$ and $X_{i}^{*}$ have finite means. This hypothesis may be relaxed by considering the dispersive order (see Rem. 4.2).

Theorem 4.1. We assume that there is no interaction, i.e. (4.1) is satisfied. Let $X_{i}^{*}$ be a random variable independent of $X^{-i}$ and assume that $X_{i}^{*} \leq e w X_{i}$ and $-\infty<\ell_{*} \leq \ell$, where $\ell_{*}$ and $\ell$ are the left-end points of the support of $X_{i}^{*}$ and $X_{i}$. If $g_{i}$ is a non-decreasing convex function, then $S_{i}^{*} \leq S_{i}$ and $S_{j}^{*} \geq S_{j}$ for $j \neq i$.

The proof of Theorem 4.1 is postponed in Appendix B.

Remark 4.2. Note that, from Proposition A.3, the previous result also holds for any non-decreasing convex or non-increasing concave function $g_{i}$ as soon as $X_{i}^{*} \leq_{\operatorname{disp}} X_{i}$ and $X_{i}^{*} \leq_{\mathrm{st}} X_{i}$. In addition, it is shown in [23] that if $X_{i}^{*} \leq_{\operatorname{disp}} X_{i}$ with common and finite left end points of their support (i.e., $\ell^{*}=\ell$ ) then $X_{i}^{*} \leq_{\text {st }} X_{i}$.

\subsection{Relation with stochastic orders in presence of interactions}

In the case where there are interactions, we have to consider the total Sobol indices as defined by (2.4). We will first show that the $i$ th total Sobol indices are ordered if $X_{i}^{*} \leq_{\operatorname{disp}} X_{i}$ and $X_{i}^{*} \leq_{\mathrm{st}} X_{i}$, provided that the function $f$ is a product of functions of one variable whose $\log$ is non-decreasing and convex; indeed, we shall 
prove that for any $\alpha \subset\{1, \ldots, k\}$ with $i \in \alpha$, if $X_{i}^{*} \leq_{\text {disp }} X_{i}$ and $X_{i}^{*} \leq_{\text {st }} X_{i}$ then, $\alpha$-Sobol indices are ordered. Then we consider some extensions of that case. Recall that, for $\alpha \subset\{1, \ldots, k\}$, the $\alpha$-Sobol index is defined as:

$$
S_{\alpha}=\frac{\operatorname{Var}\left(f_{\alpha}\left(X_{\alpha}\right)\right)}{\operatorname{Var}(Y)}
$$

Theorem 4.3. We assume that $f$ writes:

$$
f\left(X_{1}, \ldots, X_{k}\right)=g_{1}\left(X_{1}\right) \times \cdots \times g_{k}\left(X_{k}\right)+K,
$$

where $K \in \mathbb{R}$ and $g_{j}, j=1, \ldots, k$ are real-valued functions. Let $X_{i}^{*}$ be a random variable independent of $X^{-i}$ and assume that $X_{i}^{*} \leq_{\text {disp }} X_{i}$ and $X_{i}^{*} \leq_{s t} X_{i}$. If $\log g_{i}$ is a non-decreasing convex or a non-increasing concave function, then

(1) for any $\alpha \subset\{1, \ldots, k\}$ with $i \in \alpha$, we have $S_{\alpha}^{*} \leq S_{\alpha}$ and for any $\beta \subset\{1, \ldots, k\}$ with $i \notin \beta$, we have $S_{\beta}^{*} \geq S_{\beta}$,

(2) $S_{T_{i}}^{*} \leq S_{T_{i}}$.

The proof of Theorem 4.3 is postponed in Appendix B. The conditions on stochastic ordering between $X_{i}$ and $X_{i}^{*}$, and on the log-convexity are necessary, as can be seen with the two counter-examples below.

Example 4.4. To see the necessity of the stochastic ordering, one can consider:

$$
f\left(X_{1}, X_{2}, X_{3}\right)=\exp \left(\exp \left(X_{1}\right)\right) \exp \left(X_{2}\right) \exp \left(X_{3}\right),
$$

with $X_{1}, X_{2}$ and $X_{3}$ uniform on [0,1]. If $X_{1}^{*}$ is uniform on [1,1.9], then $X_{1}^{*} \leq_{\operatorname{disp}} X_{1}$ but $X_{1} \leq_{\mathrm{st}} X_{1}^{*}$ and it can be easily checked that $S_{T_{1}}^{*}>S_{T_{1}}\left(S_{T_{1}}^{*} \approx 0.90\right.$ and $\left.S_{T_{1}} \approx 0.65\right)$.

Example 4.5. The log-convexity of $g_{i}$ is also necessary. Indeed, take:

$$
f\left(X_{1}, X_{2}, X_{3}\right)=g_{1}\left(X_{1}\right) X_{2} X_{3},
$$

where

$$
g_{1}(x)= \begin{cases}0 & \text { if } x<0.45 \\ x / 10 & \text { if } 0.45 \leq x \leq 0.5 \\ x & \text { else }\end{cases}
$$

and $X_{2}, X_{3}$ uniform on [2,3], $X_{1}$ uniform on [0,1], $X_{1}^{*}$ uniform on $[0,0.5]$ so that $X_{1}^{*} \leq_{\text {disp }} X_{1}, X_{1}^{*} \leq_{\text {st }} X_{1}$ but $g_{1}$ is not log-convex. In that case, we have $S_{T_{1}}^{*}>S_{T_{1}}\left(S_{T_{1}}^{*} \approx 0.99\right.$ and $\left.S_{T_{1}} \approx 0.97\right)$.

Now, we turn to the case where $f$ writes as a sum of product of convex non-decreasing functions of one variable, that is, there are a finite set $A$ and convex non-decreasing functions $g_{i}^{a}(i \in\{1, \ldots, k\}, a \in A$,$) such$ that

$$
f(X)=\sum_{a \in A} g_{1}^{a}\left(X_{1}\right) \times \cdots \times g_{k}^{a}\left(X_{k}\right)
$$


Proposition 4.6. Assume that $f$ satisfies (4.5). Then, for any $i \in\{1, \ldots, k\}$,

$$
\begin{aligned}
f_{i}\left(X_{i}\right) & =\sum_{a \in A}\left[\left(g_{i}^{a}\left(X_{i}\right)-\mathbb{E}\left(g_{i}^{a}\left(X_{i}\right)\right) \prod_{j \neq i} \mathbb{E}\left(g_{j}^{a}\left(X_{j}\right)\right)\right],\right. \\
f_{T_{i}}(X) & =\sum_{a \in A}\left[\left(g_{i}^{a}\left(X_{i}\right)-\mathbb{E}\left(g_{i}^{a}\left(X_{i}\right)\right)\right) \prod_{j \neq i} g_{j}^{a}\left(X_{j}\right)\right], \\
\operatorname{Var}\left(f_{T_{i}}\right) & =\sum_{a, b \in A} \operatorname{Cov}\left(g_{i}^{a}\left(X_{i}\right), g_{i}^{b}\left(X_{i}\right)\right) \prod_{j \neq i} \mathbb{E}\left(g_{j}^{a}\left(X_{j}\right) g_{j}^{b}\left(X_{j}\right)\right) .
\end{aligned}
$$

Proof. The proof uses in a straightforward way the computations done in the proof of Theorem 4.3.

We deduce the two following extensions of Theorem 4.3.

Proposition 4.7. Let $\left\{I_{a}\right\}_{a \in A}$ be a partition of $\{1, \ldots, k\}$ and assume that

$$
f(X)=\sum_{a \in A} \prod_{j \in I_{a}} g_{j}\left(X_{j}\right)
$$

where the $g_{j}$ 's are real-valued functions. Let $X_{i}^{*}$ be a random variable independent of $X$ and assume that $X_{i}^{*} \leq_{\text {disp }} X_{i}$ and $X_{i}^{*} \leq_{s t} X_{i}$. If $\log g_{i}$ is a non-decreasing convex function or a non-increasing concave function, then $S_{T_{i}}^{*} \leq S_{T_{i}}$.

Proof. Given that the $I_{a}$ are disjoint, this result directly follows from the proof of Theorem 4.3.

Remark 4.8. As in Theorem 4.3, Proposition 4.7 may be generalized to the $\alpha$-Sobol indices defined in (4.3).

Proposition 4.9. Let $f(X)=\varphi_{1}\left(X_{i}\right) \prod_{j \neq i} g_{j}\left(X_{j}\right)+\varphi_{2}\left(X_{i}\right)$ with $\log \varphi_{1}$ and $\log \varphi_{2}$ non-decreasing and convex. If

- $X_{i}^{*}$ is independent of $X, X_{i}^{*} \leq_{\text {disp }} X_{i}$ and $X_{i}^{*} \leq_{s t} X_{i}$.

- $\frac{\operatorname{Var}\left(\varphi_{2}\left(X_{i}^{*}\right)\right)}{\mathbb{E}\left(\varphi_{1}\left(X_{i}^{*}\right)\right)^{2}} \leq \frac{\operatorname{Var}\left(\varphi_{2}\left(X_{i}\right)\right)}{\mathbb{E}\left(\varphi_{1}\left(X_{i}\right)\right)^{2}}$ and $\frac{\operatorname{Cov}\left(\varphi_{1}\left(X_{i}^{*}\right), \varphi_{2}\left(X_{i}^{*}\right)\right)}{\mathbb{E}\left(\varphi_{1}\left(X_{i}^{*}\right)\right)^{2}} \leq \frac{\operatorname{Cov}\left(\varphi_{1}\left(X_{i}\right), \varphi_{2}\left(X_{i}\right)\right)}{\mathbb{E}\left(\varphi_{1}\left(X_{i}\right)\right)^{2}}$.

Then $S_{T_{i}}^{*} \leq S_{T_{i}}$.

The proof is postponed to Appendix B. The second condition in Proposition 4.9 is very technical and unsatisfactory. Nevertheless, very simple counter-examples exist as it can be seen below.

Example 4.10. Let $X_{i} \sim \mathcal{U}([1.5,3.5]), X_{i}^{*} \sim \mathcal{U}([0,1.8]), \varphi_{1}(x)=\exp \left(x^{2}\right)$ and $\varphi_{2}(x)=\exp (x), g_{j}(x)=1, j \neq i$. Then one can show that $X_{i}^{*} \leq_{\operatorname{disp}} X_{i}$ and $X_{i}^{*} \leq_{\text {st }} X_{i}$. However,

$$
\begin{aligned}
\frac{\operatorname{Var}\left(\varphi_{2}\left(X_{i}^{*}\right)\right)}{\mathbb{E}\left(\varphi_{1}\left(X_{i}^{*}\right)\right)^{2}} & \sim 0.08 \\
\frac{\operatorname{Var}\left(\varphi_{2}\left(X_{i}\right)\right)}{\mathbb{E}\left(\varphi_{1}\left(X_{i}\right)\right)^{2}} & \sim 10^{-7} \\
\frac{\operatorname{Cov}\left(\varphi_{1}\left(X_{i}^{*}\right), \varphi_{2}\left(X_{i}^{*}\right)\right)}{\mathbb{E}\left(\varphi_{1}\left(X_{i}^{*}\right)\right)^{2}} & \sim 0.3 \\
\frac{\operatorname{Cov}\left(\varphi_{1}\left(X_{i}\right), \varphi_{2}\left(X_{i}\right)\right)}{\mathbb{E}\left(\varphi_{1}\left(X_{i}\right)\right)^{2}} & \sim 10^{-3},
\end{aligned}
$$


and, using computations made in the proof of Proposition 4.9, $S_{T_{i}}^{*}>S_{T_{i}}$.

The following result derived in [9] is mentioned here as a related result on excess wealth orders, even if it is not sufficient to obtain a more general version of Proposition 4.9.

Proposition 4.11 ([9], Cor. 3.2). Let $X$ and $Y$ be two random variables with finite means and supports bounded from below by $\ell_{X}$ and $\ell_{Y}$ respectively. If $X \leq$ ew $Y$ and $\ell_{X} \leq \ell_{Y}$ then for all non-decreasing and convex functions $h_{1}, h_{2}$ for which $h_{i}(X)$ and $h_{i}(Y) i=1,2$ have second order moments,

$$
\operatorname{Cov}\left(h_{1}(X), h_{2}(X)\right) \leq \operatorname{Cov}\left(h_{1}(Y), h_{2}(Y)\right) .
$$

\section{EXAMPLES}

In this section, we illustrate the previous results on some classical financial risk models. All considered models are associated with a set of parameters. Let us first recall the conditions under which some particular distribution functions are ordered with respect to the dispersive order. We refer to [17] for other classes of distribution functions.

Proposition 5.1. Let $X$ and $Y$ be two random variables.

(1) If $X \sim \mathcal{U}[a, b]$ and $Y \sim \mathcal{U}[c, d]$, then $X$ is smaller than $Y$ for the dispersive order $\left(X \leq_{\text {disp }} Y\right)$ if and only if

$$
b-a \leq d-c .
$$

(2) If $X \sim \mathcal{E}(\mu)$ and $Y \sim \mathcal{E}(\lambda)$, then $X$ is smaller than $Y$ for the dispersive order $\left(X \leq_{\text {disp }} Y\right)$ if and only if

$$
\lambda \leq \mu
$$

(3) If $X \sim \mathcal{N}\left(m_{1}, \sigma^{2}\right)$ and $Y \sim \mathcal{N}\left(m_{2}, \nu^{2}\right)$, then $X$ is smaller than $Y$ for the dispersive order $\left(X \leq_{\text {disp }} Y\right)$ if and only if

$$
\sigma \leq \nu
$$

As mentioned in Section 3, most of the numerical illustrations will be based on model parameter with truncated distribution functions. We present some properties of such distributions.

Proposition 5.2. Let $X$ and $Y$ be two random variables

(1) if $X \sim \mathcal{N}_{T}\left(m, \sigma^{2}\right)$ where $X$ is truncated on $[a, b]$ then the quantile function of $X$ is given by

$$
F_{X}^{-1}(x)=\phi^{-1}(\phi(\alpha)+x(\phi(\beta)-\phi(\alpha))) \sigma+m
$$

where $\alpha=\frac{a-m}{\sigma}, \beta=\frac{b-m}{\sigma}$ and where $\phi$ is the standard normal cumulative distribution function.

(2) if $Y \sim \mathcal{E}_{T}(\lambda)$ is truncated on $[a, b]$ then the quantile function of $Y$ is given by

$$
F_{Y}^{-1}(x)=-\frac{1}{\lambda} \log \left(e^{-\lambda a}+x\left(e^{-\lambda b}-e^{-\lambda a}\right)\right)
$$

where $\lambda$ denotes the parameter of the exponential distribution.

The following lemma gives some conditions that ensure the ordering of two truncated random variables with respect to the dispersive order. 
Lemma 5.3. Let $X$ and $Y$ be two random variables.

(1) If $X \sim \mathcal{U}[a, b]$ and $Y \sim \mathcal{N}_{T}\left(m, \sigma^{2}\right)$ where $Y$ is truncated on $[c, d]$, then $X$ is smaller than $Y$ for the dispersive order $X \leq_{\operatorname{disp}} Y$ if and only if

$$
b-a \leq \sigma \sqrt{2 \pi}(\phi(\beta)-\phi(\alpha))
$$

where $\phi$ represents the cumulative distribution of a standard Gaussian law and where $\alpha$ and $\beta$ are given as in Proposition 5.2.

(2) If $X \sim \mathcal{E}_{T}(\mu)$ and $Y \sim \mathcal{E}_{T}(\lambda)$ are truncated on the same interval then $X \leq_{\text {disp }} Y$ if and only if

$$
\lambda \leq \mu
$$

Proof. The previous conditions can be easily derived by differentiating the difference of the quantile functions $F_{Y}^{-1}-F_{X}^{-1}$ and by using the fact that this derivative should be positive.

Also, we recall (see Rem. 4.2) that if two random variables ordered for the dispersive order have the same finite left point of their support, then they are ordered for the stochastic order. In the example that we will consider, $\mathcal{N}_{T}$ (resp. $\mathcal{E}_{T}$ ) denotes the truncated Gaussian (resp. exponential) distribution on $[0,2]$.

\subsection{Value at Risk sensitivity analysis}

In Section 3, we presented the Value at Risk in a simple and classical financial model. Table 1 illustrates the consistency of total Sobol indices when the distributions of input parameters are ordered with respect to the dispersive order.

Indeed, each line of Table 1 corresponds to a scenario where one of the parameter has been increased with respect to both the dispersive and the stochastic order. As can be seen, changing the laws of model parameters $\mu$ and $\sigma$ have a significant impact on the values of total Sobol indices $S_{T_{\mu}}$ and $S_{T_{\sigma}}$. Note that the ordering among Sobol indices is fully consistent with the one predicted by Theorem 4.3.

\subsection{Vasicek model}

In risk management, present values of financial or insurance products are computed by discounting future cash-flows. In market practice, discounting is done by using the current yield curve, which gives the offered interest rate as a function of the maturity (time to expiration) for a given type of debt contract. In the Vasicek model, the yield curve is given as an output of an instantaneous spot rate model with the following risk-neutral dynamics

$$
\mathrm{d} r_{t}=a\left(b-r_{t}\right) \mathrm{d} t+\sigma \mathrm{d} W_{t}
$$

where $a, b$ and $\sigma$ are positive constants and where $W$ is a standard brownian motion. Parameter $\sigma$ is the volatility of the short rate process, $b$ corresponds to the long-term mean-reversion level whereas $a$ is the speed of convergence of the short rate process $r$ towards level $b$. The price at time $t$ of a zero coupon bond with maturity $T$ in such a model is given by (see, e.g., [5]):

$$
P(t, T)=A(t, T) e^{-r_{t} B(t, T)},
$$

where

$$
A(t, T)=\exp \left(\left(b-\frac{\sigma^{2}}{2 a^{2}}\right)(B(t, T)-(T-t))-\frac{\sigma^{2}}{4 a} B^{2}(t, T)\right)
$$


TABLE 2. Total Sobol indices as a result of a risk perturbation of $b$. All digits are significant with a $95 \%$ probability.

\begin{tabular}{llllll}
\hline Parameter & Law & Total index & Parameter & Law & Total index \\
\hline$a$ & $\mathcal{U}[0,1]$ & 0.41 & $a$ & $\mathcal{U}[0,1]$ & 0.48 \\
$b^{*}$ & $\mathcal{U}[0,1]$ & 0.52 & $b$ & $\mathcal{U}[0,2]$ & 0.57 \\
$\sigma$ & $\mathcal{U}[0,1]$ & 0.18 & $\sigma$ & $\mathcal{U}[0,1]$ & 0.06 \\
\hline
\end{tabular}

TABLE 3. Total Sobol indices as a result of a risk perturbation of $\sigma$. All digits are significant with a $95 \%$ probability.

\begin{tabular}{llllll}
\hline Parameter & Law & Total index & Parameter & Law & Total index \\
\hline$a$ & $\mathcal{U}[0,1]$ & 0.41 & $a$ & $\mathcal{U}[0,1]$ & 0.25 \\
$b$ & $\mathcal{U}[0,1]$ & 0.52 & $b$ & $\mathcal{U}[0,1]$ & 0.13 \\
$\sigma^{*}$ & $\mathcal{U}[0,1]$ & 0.18 & $\sigma$ & $\mathcal{N}_{\mathrm{T}}(0.5,2)$ & 0.70 \\
\hline
\end{tabular}

and

$$
B(t, T)=\frac{1-e^{-a(T-t)}}{a}
$$

The yield-curve can be obtained as a deterministic transformation of zero-coupon bond prices at different maturities.

In what follows, we quantify the relative importance of the input parameters $\{a, b, \sigma\}$ affecting the uncertainty in the zero-coupon bond price at time $t=0$. In the following numerical experiments, the maturity $T$ and the initial spot rate $r_{0}$ are chosen such that $T=1$ and $r_{0}=10 \%$. Tables 2 and 3 report the total Sobol indices of the parameter $a, b, \sigma$ under two different risk perturbations in the probability laws of these parameters. Table 2 illustrates the effect of an increase of the mean-reverting level with respect to the dispersive order and the stochastic dominance order, i.e., $b^{*} \leq_{\text {disp }} b$ and $b^{*} \leq_{\text {st }} b$. We observe from Table 2 that the relative importance of the mean-reverting level $b$ increases from 0.52 to 0.57. Although the total Sobol index of $\sigma$ decreases, the total index of $a$ increases. Note that the assumptions of Theorem 4.3 are not satisfied here: one can show that the output function (5.2) is $\log$ non-decreasing and log convex in $b$ but the multiplicative form (4.4) does not hold.

Table 3 displays the total Sobol indices when $\sigma^{*} \leq_{\operatorname{disp}} \sigma$ and $\sigma^{*} \leq_{\text {st }} \sigma$. The law of $\sigma$ is taken as a truncated Gaussian random variable on $[0,2]$ and has a variance of 0.3 . We observe an increase in the total Sobol index of $\sigma$ and a decrease in total index of $a$ and $b$.

\subsection{Heston model}

In finance, the Heston model is a mathematical model which assumes that the stock price $S_{t}$ has a stochastic volatility $\sigma_{t}$ that follows a CIR process. The model is represented by the following bivariate system of stochastic differential equations (SDEs) (see, e.g., [10])

$$
\begin{aligned}
& \mathrm{d} S_{t}=(r-q) S_{t} \mathrm{~d} t+\sqrt{\sigma_{t}} S_{t} \mathrm{~d} B_{t} \\
& \mathrm{~d} \sigma_{t}=\kappa\left(\theta-\sigma_{t}\right) \mathrm{d} t+\sigma \sqrt{\sigma_{t}} \mathrm{~d} W_{t},
\end{aligned}
$$

where $\mathrm{d}\langle B, W\rangle_{t}=\rho \mathrm{d} t$. 
The model parameters are

- $r$ : the risk-free rate,

- $q$ : the dividend rate,

- $\kappa>0$ : the mean reversion speed of the volatility,

- $\theta>0$ : the mean reversion level of the volatility,

- $\sigma>0$ : the volatility of the volatility,

- $\sigma_{0}>0$ : the initial level of volatility,

- $\rho \in[-1,1]$ : the correlation between the two Brownian motions $B$ and $W$.

The numerical computation of European option prices under this model can be done by using the fast Fourier transform approach developed in [7] which is applicable when the characteristic function of the logarithm of $S_{t}$ is known in a closed form. In this framework, the price at time $t$ of a European call option with strike $K$ and time to maturity $T$ is given by

$$
C(t, K, T)=S_{t} e^{-q \tau} P_{1}-K e^{-r \tau} P_{2}
$$

where for $j=1,2$

$$
\begin{aligned}
P_{j} & =\frac{1}{2}+\frac{1}{\pi} \int_{0}^{\infty} \Re\left(\frac{e^{-i \phi \log K} f_{j}\left(\phi ; x_{t}, \sigma_{t}\right)}{i \phi}\right) \mathrm{d} \phi \\
f_{j}\left(\phi ; x_{t}, \sigma_{t}\right) & =\exp \left(C_{j}(\phi ; \tau)+D_{j}(\phi ; \tau) \sigma_{t}+i \phi x_{t}\right) \\
C_{j} & =(r-q) i \phi \tau+\frac{\kappa \theta}{\sigma^{2}}\left[\left(b_{j}-\rho \sigma i \phi+d_{j}\right) \tau-2 \log \left(\frac{1-g_{j} e^{d_{j} \tau}}{1-g_{j}}\right)\right] \\
D_{j} & =\frac{b_{j}-\rho \sigma i \phi+d_{j}}{\sigma^{2}}\left(\frac{1-e^{d_{j} \tau}}{1-g_{j} e^{d_{j} \tau}}\right) \\
g_{j} & =\frac{b_{j}-\rho \sigma i \phi+d_{j}}{b_{j}-\rho \sigma i \phi-d_{j}} \\
d_{j} & =\sqrt{\left(\rho \sigma i \phi-b_{j}\right)^{2}-\sigma^{2}\left(2 u_{j} i \phi-\phi^{2}\right)} \\
u_{1} & =\frac{1}{2}, u_{2}=-\frac{1}{2}, b_{1}=\kappa-\rho \sigma, b_{2}=\kappa, x_{t}=\log S_{t}, \tau=T-t .
\end{aligned}
$$

Given that the input parameter are not known with certainty, which one mostly affect the uncertainty of the output pricing function (5.5). Table 4 displays the total Sobol indices of each parameter under two assumptions on the distribution of input parameter. In the first case ( 3 first columns), all parameters are assumed to be uniformly distributed. In the second case (3 last columns), we only change the distribution of the interest rate parameter $r$ is such as way that $r^{*} \leq_{\operatorname{disp}} r$ and $r^{*} \leq_{\text {st }} r$. The option characteristics are taken as follows: $T=0.5$, $S_{0}=100, K=100$.

As can be observed in Table 4, the influence of the input factors $\kappa, \theta, \sigma$ and $\rho$ is negligible under the two considered assumptions. Note that most of the uncertainty in the option price is due to the dividend yield $q$ and the interest rate $r$. Similar conclusions are outlined in [21] but the difference here is that we analyze how the total Sobol indices are affected by a change in the law of some input parameters. Interestingly, when the law of the interest rate parameter $r^{*}$ is changed to $r$, this parameter becomes more important than the dividend yield in terms of output uncertainty.

\section{Multivariate extension}

Here we present some multivariate extensions of the previous results. Our goal is to explore how an increase of riskness (in the sense of multivariate stochastic orders) of a group of input parameters may have an impact 
TABLE 4. Total Sobol indices for the price of a call option in the Heston model. All digits are significant with a $95 \%$ probability.

\begin{tabular}{llllll}
\hline Parameter & Law & Total index & Parameter & Law & Total index \\
\hline$r^{*}$ & $\mathcal{U}[0,1]$ & 0.32 & $r$ & $\mathcal{U}[0,2]$ & 0.73 \\
$q$ & $\mathcal{U}[0,1]$ & 0.39 & $q$ & $\mathcal{U}[0,1]$ & 0.20 \\
$\kappa$ & $\mathcal{U}[0,1]$ & 0.0036 & $\kappa$ & $\mathcal{U}[0,1]$ & 0.0009 \\
$\theta$ & $\mathcal{U}[0,1]$ & 0.0082 & $\theta$ & $\mathcal{U}[0,1]$ & 0.0020 \\
$\sigma$ & $\mathcal{U}[0,1]$ & 0.0012 & $\sigma$ & $\mathcal{U}[0,1]$ & 0.0004 \\
$\sigma_{0}$ & $\mathcal{U}[0,1]$ & 0.30 & $\sigma_{0}$ & $\mathcal{U}[0,1]$ & 0.08 \\
$\rho$ & $\mathcal{U}[0,1]$ & 0.0011 & $\rho$ & $\mathcal{U}[0,1]$ & 0.0004 \\
\hline
\end{tabular}

on the output. We shall be mainly interested in the multivariate dispersive order and the univariate stochastic increasing convex order. We refer to [22] for a detailed review on the subject.

\subsection{Multivariate stochastic order and impact on Sobol indices}

Let $X=\left(X_{1}, \ldots, X_{n}\right)$ be a random vector with joint distribution function $\mathrm{F}$. Denote by $F_{1}$ the marginal distribution function of $X_{1}$. Consider $u=\left(u_{1}, \ldots, u_{n}\right) \in(0,1)^{n}$ and define

$$
x_{1}\left(u_{1}\right)=F_{1}^{-1}\left(u_{1}\right)
$$

then define recursively

$$
x_{i}\left(u_{1}, \ldots, u_{i}\right)=F_{i \mid 1, \ldots, i-1}^{-1}\left(u_{i} \mid x_{1}, \ldots, x_{i-1}\right), i=2, \ldots, n,
$$

where $F_{i \mid 1, \ldots, i-1}$ is the conditional distribution function of $X_{i}$ given that $\left(X_{1}=x_{1}, \ldots, X_{i-1}=x_{i-1}\right), i=$ $2,3 \ldots, n$.

In the same way, for another random vector $Y=\left(Y_{1}, \ldots, Y_{n}\right)$ with joint distribution function $G$, define

$$
y_{1}\left(u_{1}\right)=G_{1}^{-1}\left(u_{1}\right)
$$

then define recursively

$$
y_{i}\left(u_{1}, \ldots, u_{i}\right)=G_{i \mid 1, \ldots, i-1}^{-1}\left(u_{i} \mid y_{1}, \ldots, y_{i-1}\right), i=2, \ldots, n
$$

$G_{i \mid 1, \ldots, i-1}$ is the conditional distribution function of $Y_{i}$ given that $\left(Y_{1}=y_{1}, \ldots, Y_{i-1}=y_{i-1}\right), i=2,3 \ldots, n$.

Definition 6.1. We say that $X$ is smaller than $Y$ for the multivariate dispersive order, denoted by $X \leq{ }_{\operatorname{Disp}} Y$, if $y_{i}(u)-x_{i}(u)$ is componentwise increasing in $u=\left(u_{1}, \ldots, u_{i}\right) \in(0,1)^{i}$ for $i=1, \ldots, n$, where $x_{i}(u)$ and $y_{i}(u)$ are defined respectively in (6.2) and (6.4).

Definition 6.2. Let $X$ and $Y$ be two one dimensional random variables. We say that $X$ is smaller than $Y$ in the st:icx order, denoted by $X \leq_{\text {st:icx }} Y$, if

$$
\mathbb{E}(\phi(X)) \leq \mathbb{E}(\phi(Y))
$$

for all increasing function $\phi$, and if

$$
\operatorname{Var}(\phi(X)) \leq \operatorname{Var}(\phi(Y))
$$


for all increasing convex function $\phi$.

Definition 6.3. In what follows, $\leq$ stands for the coordinatewise ordering on $\mathbb{R}^{n}$ and we use the notation $[x, y] \leq z$ for any $x, y, z \in \mathbb{R}^{n}$ such that $x \leq z$ and $y \leq z$ (similarly, $z \leq[x, y]$ if $z \leq x$ and $z \leq y$ ). A real function $\phi$ on $\mathbb{R}^{n}$ is said to be directionally convex if for any $x_{i} \in \mathbb{R}^{n}, i=1,2,3,4$ such that $x_{1} \leq\left[x_{2}, x_{3}\right] \leq x_{4}$ and $x_{1}+x_{4}=x_{2}+x_{3}$, one has

$$
\phi\left(x_{2}\right)+\phi\left(x_{3}\right) \leq \phi\left(x_{1}\right)+\phi\left(x_{4}\right)
$$

A real function $\phi$ on $\mathbb{R}^{n}$ is said to be directionally concave if, under the same condition, one has

$$
\phi\left(x_{2}\right)+\phi\left(x_{3}\right) \geq \phi\left(x_{1}\right)+\phi\left(x_{4}\right)
$$

The following result proved in [22] gives conditions under which functions of two random vectors are ordered according to the st:icx order.

Proposition 6.4. [22] Let $X$ and $Y$ be two $n$-dimensional nonnegative independent random vectors. If $X \leq$ Disp $Y$, then

$$
\phi(X) \leq_{s t: i c x} \phi(Y)
$$

for all increasing directionally convex functions $\phi: \mathbb{R}^{n} \rightarrow \mathbb{R}$

As before, we consider a model output of the form $Y=f\left(X_{1}, \ldots, X_{k}\right)$ where $X_{1}, \ldots, X_{k}$ are independent random variables. For $u \subset\{1, \ldots, k\}$, we denote by $X_{u}$ the random vector $\left(X_{i}, i \in u\right), X_{-u}=\left(X_{i}, i \notin u\right)$ and $|u|$ denotes the length of $u$, i.e. its number of elements. Thus, the impact of a group of variables $X_{u}, u \subset\{1, \ldots, k\}$ on $Y=f(X)$ may be measured by the total Sobol index (see, e.g., [12])

$$
S_{T_{u}}=1-\frac{\operatorname{Var}\left(\mathbb{E}\left(f(X) \mid X_{-u}\right)\right)}{\operatorname{Var}(Y)}
$$

Using the variance decomposition formula, (6.5) can be equivalently written as

$$
S_{T_{u}}=\frac{\mathbb{E}\left(\operatorname{Var}\left(f(X) \mid X_{-u}\right)\right)}{\operatorname{Var}(Y)}
$$

\subsection{Relationship with multivariate stochastic order: the additive case}

In this subsection we suppose that $f(X)$ can be expressed in the following additive form, i.e for all non empty set $u \subset\{1, \ldots, k\}$

$$
f\left(X_{1}, \ldots, X_{k}\right)=g\left(X_{u}\right)+h\left(X_{-u}\right)+K
$$

where $g: \mathbb{R}^{|u|} \rightarrow \mathbb{R}$ and $h: \mathbb{R}^{|-u|} \rightarrow \mathbb{R}$ are real valued functions and $K \in \mathbb{R}$.

As in the univariate case, for $u \subset\{1, \ldots, k\}, X_{u}^{*}$ denotes another random vector that will be compared to $X_{u}$. We suppose that for non empty $u \subset\{1, \ldots, k\}$, the vector of input parameters $X$ can be represented as $X=\left(X_{u}, X_{-u}\right)$. We shall assume $X_{u}^{*} \leq_{\text {Disp }} X_{u}$ and study the impact of replacing the group of variable $X_{u}$ by $X_{u}^{*}$ on total Sobol indices. We assume that $X_{u}^{*}$ is independent of $X_{-u}$ and we denote by $X^{*}=\left(X_{u}^{*}, X_{-u}\right)$ the 
vector $X$ where $X_{u}$ has been replaced by $X_{u}^{*}$ and by

$$
S_{T_{u}}^{*}=\frac{\mathbb{E}\left(\operatorname{Var}\left(f\left(X^{*}\right) \mid X_{-u}\right)\right)}{\operatorname{Var}\left(Y^{*}\right)} .
$$

the total Sobol indices of $X_{u}^{*}$ associated to the output $Y^{*}=f\left(X^{*}\right)$.

Theorem 6.5. We assume that (6.7) is satisfied. Let $X_{u}^{*}$ be a random vector independent of $X_{-u}$ and such that $X_{u}^{*} \leq{ }_{\text {Disp }} X_{u}$. If $g$ is a non-decreasing directionally convex function, then $S_{T_{u}}^{*} \leq S_{T_{u}}$.

The proof of Theorem 6.5 makes use of Proposition 6.4.

Proof. The proof makes uses in a straightforward way of the computations done in the proof of Theorem 4.1.

\subsection{Relationship with multivariate stochastic order: the product case}

Here, we consider the extensions of the previous result in the case where the function $f$ is a product of functions with several variables which fulfill some multivariate monotonicity conditions.

Theorem 6.6. We assume that for a non empty set $u \subset\{1, \ldots, k\}$, the output function $f$ writes

$$
f\left(X_{1}, \ldots, X_{k}\right)=g\left(X_{u}\right) \times h\left(X_{-u}\right)+K
$$

where $g: \mathbb{R}^{|u|} \rightarrow \mathbb{R}$ and $h: \mathbb{R}^{|-u|} \rightarrow \mathbb{R}$ are real valued functions and $K \in \mathbb{R}$. Let $X_{u}^{*}$ be a random vector independent of $X_{-u}$ and assume that $X_{u}^{*} \leq_{\text {Disp }} X_{u}$. If $g$ is a non-increasing directionally concave function, then $S_{T_{u}}^{*} \leq S_{T_{u}}$.

Proof. Remember that

$$
S_{T_{u}}=\frac{\mathbb{E}\left(\operatorname{Var}\left(f(X) \mid X_{-u}\right)\right)}{\operatorname{Var}(Y)}
$$

By definition

$$
\begin{aligned}
\mathbb{E}\left(\operatorname{Var}\left(f(X) \mid X_{-u}\right)\right) & =\mathbb{E}\left[f(X)-\mathbb{E}\left(f(X) \mid X_{-u}\right)\right]^{2} \\
& =\mathbb{E}\left[h\left(X_{-u}\right)\left(g\left(X_{u}\right)-\mathbb{E}\left(g\left(X_{u}\right)\right)\right)\right]^{2} \\
& =\mathbb{E} h^{2}\left(X_{-u}\right) \operatorname{Var}\left(g\left(X_{u}\right)\right)
\end{aligned}
$$

since every measurable function of $X_{u}$ and $X_{-u}$ are independent (because $X_{u}$ and $X_{-u}$ are supposed to be independent random vector ). Thus using the variance decomposition formula, (6.10) can be equivalently written as

$$
S_{T_{u}}=\left[1+\frac{\operatorname{Var}\left(h\left(X_{-u}\right)\right)\left[\mathbb{E}\left(g\left(X_{u}\right)\right)\right]^{2}}{\operatorname{Var}\left(g\left(X_{u}\right)\right) \mathbb{E} h^{2}\left(X_{-u}\right)}\right]^{-1} .
$$

With the same reasoning, one can show that

$$
S_{T_{u}}^{*}=\left[1+\frac{\operatorname{Var}\left(h\left(X_{-u}\right)\right)\left[\mathbb{E}\left(g\left(X_{u}^{*}\right)\right)\right]^{2}}{\operatorname{Var}\left(g\left(X_{u}^{*}\right)\right) \mathbb{E} h^{2}\left(X_{-u}\right)}\right]^{-1} .
$$


Hence the result holds if

$$
\frac{\operatorname{Var}\left(g\left(X_{u}^{*}\right)\right)}{\left[\mathbb{E}\left(g\left(X_{u}^{*}\right)\right)\right]^{2}} \leq \frac{\operatorname{Var}\left(g\left(X_{u}\right)\right)}{\left[\mathbb{E}\left(g\left(X_{u}\right)\right)\right]^{2}}
$$

As $g$ is a non-increasing directionally concave function, that is $-g$ is a non-decreasing directionally convex function and thus Proposition 6.4 implies $-g\left(X_{u}^{*}\right) \leq_{\text {st:icx }}-g\left(X_{u}\right)$ which implies that $\operatorname{Var}\left(-g\left(X_{u}^{*}\right)\right) \leq \operatorname{Var}\left(-g\left(X_{u}\right)\right)$ and $\mathbb{E}\left(-g\left(X_{u}^{*}\right)\right) \leq \mathbb{E}\left(-g\left(X_{u}\right)\right)$ i.e $\mathbb{E}\left(g\left(X_{u}^{*}\right)\right) \geq \mathbb{E}\left(g\left(X_{u}\right)\right)$ which concludes the proof.

The following result proved in [22] shows an interesting closure property of the st:icx order.

Proposition 6.7. Let $X_{1}, X_{2}, \ldots, X_{n}$ a set of independent random variables, and let $Y_{1}, Y_{2}, \ldots, Y_{n}$ another set of independent random variables. If $X_{i} \leq_{\text {st:icx }} Y_{i}, i=1,2, \ldots, n$, then

$$
\phi\left(X_{1}, \ldots, X_{n}\right) \leq_{s t: i c x} \phi\left(Y_{1}, \ldots, Y_{n}\right)
$$

for all increasing directionally convex functions $\phi: \mathbb{R}^{n} \rightarrow \mathbb{R}$.

The following result extend the Theorem 6.6.

Theorem 6.8. Let

$$
f(X)=\varphi_{1}\left(X_{u}\right) h\left(X_{-u}\right)+\varphi_{2}\left(X_{u}\right)
$$

where $\varphi_{1}: \mathbb{R}^{|u|} \rightarrow \mathbb{R}, h: \mathbb{R}^{|-u|} \rightarrow \mathbb{R}$ and $\varphi_{2}: \mathbb{R}^{|u|} \rightarrow \mathbb{R}$ are real valued functions. Let $X_{u}^{*}$ be a random vector independent of $X_{-u}$ and assume that $X_{u}^{*} \leq \operatorname{Disp}_{u} X_{u}$. If $\varphi_{1}$ and $\varphi_{2}$ are decreasing and directionally concave functions, then $S_{T_{u}}^{*} \leq S_{T_{u}}$.

Proof. The proof makes use of Proposition 6.7 and involves similar computations as in the proof of Proposition 4.9 .

\section{CONCLUDING REMARKS}

We have enlightened the fact that Sobol indices are compatible with the stochastic ordering theory, and more specifically with the excess wealth order or with the dispersive order, provided that the output function satisfies some monotonicity and convexity properties. Our results have multidimensional extensions. The Vasicek and Heston model examples suggest that the hypothesis on the form of the output function $f$ might be relaxed (hypothesis of Thm. 4.3 are not fulfilled, especially in the Heston case). In other words, the compatibility between Sobol indices and stochastic orders should hold for more general functions than those considered in the present paper. On an other hand, as shown in the examples, the choice of the input laws is crucial: the most influential parameters (those associated with the greatest Sobol index) may change as some input distributions are perturbed. A way to overcome this difficulty could be to use our consistency results to generate universal bounds on Sobol indices, for a given class of input laws.

\section{Appendix A. Stochastic orders}

Let us recall some particular notions of ordering for random variables and for random vectors. We refer to $[17,23]$ or $[8]$ for a detailed review on stochastic orders, on their relationships and properties. 


\section{A.1 Definitions of stochastic orders}

We shall be mainly interested in the convex order, the dispersive order, the excess wealth order, the $*$ order and the Lorenz order. For a random variable $X$, let us denote by $F_{X}$ its distribution function, and by $F_{X}^{-1}$ the generalized inverse of $F_{X}$ (or the quantile function). The survival function of $X$ is $\bar{F}_{X}=1-F_{X}$.

Definition A.1. Let $X$ and $Y$ be two random variables, we say that

(1) $X$ is smaller than $Y$ for the standard stochastic order $\left(X \leq_{\mathrm{st}} Y\right)$ if and only if, for any bounded and non-decreasing function $f$,

$$
\mathbb{E}(f(X)) \leq \mathbb{E}(f(Y))
$$

(2) $X$ is smaller than $Y$ for the convex $\operatorname{order}\left(X \leq_{\mathrm{cx}} Y\right)$ if and only if, for any bounded convex function $f$,

$$
\mathbb{E}(f(X)) \leq \mathbb{E}(f(Y))
$$

(3) If $X$ and $Y$ have finite means, then $X$ is smaller than $Y$ for the dilatation order $\left(X \leq_{\text {dil }} Y\right)$ if and only if

$$
(X-\mathbb{E}(X)) \leq_{\mathrm{cx}}(Y-\mathbb{E}(Y))
$$

(4) $X$ is smaller than $Y$ for the dispersive order $\left(X \leq_{\operatorname{disp}} Y\right)$ if and only if $F_{Y}^{-1}-F_{X}^{-1}$ is non-decreasing.

(5) If $X$ and $Y$ have finite means, then $X$ is smaller than $Y$ for the excess wealth order $(X \leq$ ew $Y)$ if and only if for all $p \in] 0,1[$,

$$
\int_{\left[F_{X}^{-1}(p), \infty[\right.} \bar{F}_{X}(x) \mathrm{d} x \leq \int_{\left[F_{Y}^{-1}(p), \infty[\right.} \bar{F}_{Y}(x) \mathrm{d} x .
$$

(6) If $X$ and $Y$ are non negative, $X$ is smaller than $Y$ for the star order $\left(X \leq_{*} Y\right)$ if and only if

$$
\frac{F_{Y}^{-1}}{F_{X}^{-1}} \text { is non-decreasing. }
$$

(7) If $X$ and $Y$ are non negative with finite mean, $X$ is smaller than $Y$ for the Lorenz $(X \leq$ Lorenz $Y)$ if and only if

$$
\frac{X}{\mathbb{E}(X)} \leq \mathrm{cx} \frac{Y}{\mathbb{E}(Y)}
$$

Remark A.2. The $s t$ and $c x$ orders may be defined in the same way for random vectors.

\section{A.2 Some relationships between variance and stochastic orders}

It is well-known that the stochastic order and the convex order are not location-free and may not be compatible with the variance. The dispersive and excess wealth orders are location-free and in accordance with the variance. In what follows, we will consider the excess wealth order, for which we can prove the ordering of Sobol's indices. Let us remark that the dispersive order implies the excess wealth order. Natural examples of random variables ordered with respect to the dispersive order will be recalled in Section 5 . The following results proved in [23], show that the excess wealth order (and thus the dispersive order) is in accordance with the variance. 
Proposition A.3. [23] Let $X$ and $Y$ be two random variables with finite means.

(1) If $X \leq_{\text {disp }} Y$ then $X \leq{ }_{e w} Y$ then $X \leq_{d i l} Y$ and thus if $X$ and $Y$ admit an order 2 moment, $\operatorname{Var}(X) \leq$ $\operatorname{Var}(Y)$.

(2) If $X$ and $Y$ are non negative and $X \leq * Y$ then $X \leq{ }_{\text {Lorenz }} Y$ and then

$$
\frac{\operatorname{Var}(X)}{\mathbb{E}(X)^{2}} \leq \frac{\operatorname{Var}(Y)}{\mathbb{E}(Y)^{2}}
$$

(3) If $X$ and $Y$ are non negative then $X \leq * Y$ if and only if $\log X \leq{ }_{\text {disp }} \log Y$.

(4) If $X \leq{ }_{\text {disp }} Y$ and $X \leq \leq_{s t} Y$ then for all non-decreasing convex or non-increasing concave function $f$, $f(X) \leq \operatorname{disp} f(Y)$.

(5) If $X$ and $Y$ are continuous random variables with supports bounded from below by (resp.) $\ell_{*}$ and $\ell$, $X \leq e w Y$ and $-\infty<\ell_{*} \leq \ell$, then for all non-decreasing and convex function $f$, for which $f(X)$ and $f(Y)$ have finite means, we have $f(X) \leq$ ew $f(Y)$ ([24], Thm. 4.2).

Remark A.4. Result (5) above has been incorrectly stated in [13, 23], where the hypothesis on the left-end points of the supports was missing. This hypothesis is indeed required, as shown by the following example. Consider $X$ which follows a uniform law on $[1,1.9]$ and $Y$ which follows a uniform law on $[0,1]$. Then $X \leq$ ew $Y$. Let $f=\exp$ which is a convex and increasing function. We have that $\operatorname{Var}(f(X)) \sim 1.32$ and $\operatorname{Var}(f(Y)) \sim 0.24$ so that $f(X)$ cannot be less than $f(Y)$ for the ew order (see (1) of Prop. A.3). The correct statement and proof of (5) above may be found in [24], as well as an example showing that the left-end points of the support have also to be finite.

\section{Appendix B. Proofs of the main Results}

Proof of Theorem 4.1. As $g_{i}$ is a non-decreasing convex function, Proposition A.3 implies $g_{i}\left(X_{i}^{*}\right) \leq$ ew $g_{i}\left(X_{i}\right)$ and $\operatorname{Var}\left(g_{i}\left(X_{i}^{*}\right)\right) \leq \operatorname{Var}\left(g_{i}\left(X_{i}\right)\right)$. Now, the Hoeffding's decomposition of $f(X)$ can be expressed as (4.2) where $f_{\varnothing}=\mathbb{E}[f(X)]$ and $f_{j}\left(X_{j}\right)=g_{j}\left(X_{j}\right)-\mathbb{E}\left[g_{j}\left(X_{j}\right)\right]$ and the Hoeffding's decomposition of $f\left(X^{*}\right)$ writes

$$
f\left(X^{*}\right)=\sum_{j \neq i} f_{j}\left(X_{j}\right)+f_{i}^{*}\left(X_{i}^{*}\right)+f_{\varnothing}^{*}
$$

where $f_{\varnothing}^{*}=\mathbb{E}\left[f\left(X^{*}\right)\right]$ and $f_{i}^{*}\left(X_{i}^{*}\right)=g_{i}\left(X_{i}^{*}\right)-\mathbb{E}\left[g_{i}\left(X_{i}^{*}\right)\right]$.

Then, from (2.3), the $i$ th Sobol indices of $f(X)$ and $f\left(X^{*}\right)$ are such that

$$
S_{i}=\left[1+\frac{\sum_{j \neq i} \operatorname{Var}\left(g_{j}\left(X_{j}\right)\right)}{\operatorname{Var}\left(g_{i}\left(X_{i}\right)\right)}\right]^{-1}
$$

and

$$
S_{i}^{*}=\frac{\operatorname{Var}\left(g_{i}\left(X_{i}^{*}\right)\right)}{\sum_{j \neq i} \operatorname{Var}\left(g_{j}\left(X_{j}\right)\right)+\operatorname{Var}\left(g_{i}\left(X_{i}^{*}\right)\right)}
$$




$$
=\left[1+\frac{\sum_{j \neq i} \operatorname{Var}\left(g_{j}\left(X_{j}\right)\right)}{\operatorname{Var}\left(g_{i}\left(X_{i}^{*}\right)\right)}\right]^{-1} .
$$

We have already noticed that $\operatorname{Var}\left(g_{i}\left(X_{i}^{*}\right)\right) \leq \operatorname{Var}\left(g_{i}\left(X_{i}\right)\right)$ and thus we conclude that $S_{i}^{*} \leq S_{i}$. The result for $j \neq i$ follows from the fact that $\operatorname{Var}\left(g_{i}\left(X_{i}^{*}\right)\right) \leq \operatorname{Var}\left(g_{i}\left(X_{i}\right)\right)$ and

$$
S_{j}=\frac{\operatorname{Var}\left(g_{j}\left(X_{j}\right)\right)}{\sum_{j \neq i} \operatorname{Var}\left(g_{j}\left(X_{j}\right)\right)+\operatorname{Var}\left(g_{i}\left(X_{i}\right)\right)} \text { and } S_{j}^{*}=\frac{\operatorname{Var}\left(g_{j}\left(X_{j}\right)\right)}{\sum_{j \neq i} \operatorname{Var}\left(g_{j}\left(X_{j}\right)\right)+\operatorname{Var}\left(g_{i}\left(X_{i}^{*}\right)\right)} .
$$

Proof of Theorem 4.3. Without loss of generality, we may assume that $K=0$. With the hypothesis of Theorem 4.3 , the decomposition (2.1) satisfies: for all $j=1, \ldots, k$,

$$
f_{j}\left(X_{j}\right)=\left(g_{j}\left(X_{j}\right)-\mathbb{E}\left(g_{j}\left(X_{j}\right)\right) \prod_{\ell \neq j} \mathbb{E}\left(g_{\ell}\left(X_{\ell}\right)\right),\right.
$$

and following, e.g. [14], for $\alpha \subset\{1, \ldots, k\}$, we have

$$
f_{\alpha}\left(X_{\alpha}\right)=\sum_{\beta \subset \alpha}(-1)^{|\alpha|-|\beta|} \mathbb{E}\left(f(X) \mid X_{\beta}\right)
$$

The form of $f$ then gives:

$$
\begin{aligned}
f_{\alpha}\left(X_{\alpha}\right) & =\sum_{\beta \subset \alpha}(-1)^{|\alpha|-|\beta|} \prod_{j \in \beta} g_{j}\left(X_{j}\right) \prod_{j \notin \beta} \mathbb{E}\left(g_{j}\left(X_{j}\right)\right) \\
& =\prod_{j \notin \alpha} \mathbb{E}\left(g_{j}\left(X_{j}\right)\right) \prod_{j \in \alpha}\left(g_{j}\left(X_{j}\right)-\mathbb{E}\left(g_{j}\left(X_{j}\right)\right)\right)
\end{aligned}
$$

From (2.4) and (4.3), we have that

$$
S_{T_{i}}=\sum_{\substack{\alpha \subset\{1, \ldots, k\} \\ \alpha \ni i}} S_{\alpha}
$$

So that, the results for the total Sobol indices will follow from the results for $\alpha$-Sobol indices.

Equation (B.5) gives for any $\beta \subset\{1, \ldots, k\}$ :

$$
\operatorname{Var}\left(f_{\beta}\right)=\prod_{j \notin \beta} \mathbb{E}\left(g_{j}\left(X_{j}\right)\right)^{2} \prod_{j \in \beta} \operatorname{Var}\left(g_{j}\left(X_{j}\right)\right) .
$$

Moreover,

$$
\operatorname{Var}(Y)=\sum_{\beta \subset\{1, \ldots, k\}} \operatorname{Var}\left(f_{\beta}\left(X_{\beta}\right)\right)
$$


As in the proof of Theorem 4.1, $S_{\alpha}$ may be rewritten as

$$
S_{\alpha}=\left[1+\frac{\sum_{\beta \neq \alpha} \operatorname{Var}\left(f_{\beta}\left(X_{\beta}\right)\right.}{\operatorname{Var}\left(f_{\alpha}\left(X_{\alpha}\right)\right)}\right]^{-1} .
$$

Let $\alpha \subset\{1, \ldots, k\}$ with $i \in \alpha$, using (B.7),

$$
\operatorname{Var}\left(f_{\alpha}\left(X_{\alpha}\right)\right)=\operatorname{Var}\left(g_{i}\left(X_{i}\right)\right) \cdot \prod_{j \notin \alpha} \mathbb{E}\left(g_{j}\left(X_{j}\right)\right)^{2} \cdot \prod_{\substack{j \in \alpha \\ j \neq i}} \operatorname{Var}\left(g_{j}\left(X_{j}\right)\right)
$$

and

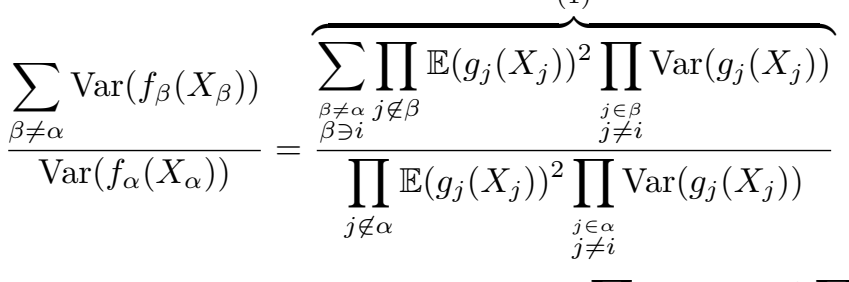

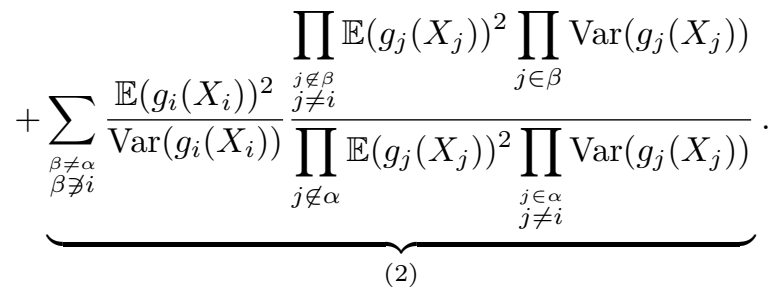

The term (1) above will not change if $X_{i}$ is replaced by $X_{i}^{*}$, neither will any product in (2). Hence, $S_{\alpha}^{*} \leq S_{\alpha}$ holds if

$$
\frac{\operatorname{Var}\left(g_{i}\left(X_{i}^{*}\right)\right)}{\mathbb{E}\left(g_{i}\left(X_{i}^{*}\right)\right)^{2}} \leq \frac{\operatorname{Var}\left(g_{i}\left(X_{i}\right)\right)}{\mathbb{E}\left(g_{i}\left(X_{i}\right)\right)^{2}}
$$

We have (see Prop. A.3)

$$
\begin{gathered}
\log g_{i}\left(X_{i}^{*}\right) \leq_{\text {disp }} \log g_{i}\left(X_{i}\right) \Longleftrightarrow g_{i}\left(X_{i}^{*}\right) \leq_{*} g_{i}\left(X_{i}\right) \\
\text { so that } g_{i}\left(X_{i}^{*}\right) \leq_{\text {Lorenz }} g_{i}\left(X_{i}\right) \Longrightarrow \frac{\operatorname{Var}\left(g_{i}\left(X_{i}^{*}\right)\right)}{\mathbb{E}\left(g_{i}\left(X_{i}^{*}\right)\right)^{2}} \leq \frac{\operatorname{Var}\left(g_{i}\left(X_{i}\right)\right)}{\mathbb{E}\left(g_{i}\left(X_{i}\right)\right)^{2}}
\end{gathered}
$$


Thus, we have proved that, if $i \in \alpha$, we have $S_{\alpha}^{*} \leq S_{\alpha}$, so that $S_{T_{i}}^{*} \leq S_{T_{i}}$ given (B.6).

When $i \notin \alpha$, using (B.8), we have:

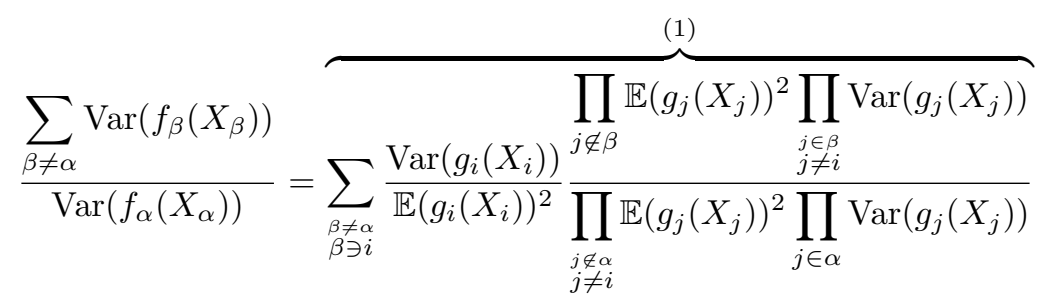

$$
\begin{aligned}
& +\underbrace{\sum_{\substack{\beta \neq \alpha \\
\beta \not i}} \frac{\prod_{\substack{j \notin \beta \\
j \neq i}} \mathbb{E}\left(g_{j}\left(X_{j}\right)\right)^{2} \prod_{j \in \beta} \operatorname{Var}\left(g_{j}\left(X_{j}\right)\right)}{\left.\prod_{j \neq i}\left(X_{j}\right)\right)^{2} \prod_{j \in \alpha} \operatorname{Var}\left(g_{j}\left(X_{j}\right)\right)}}_{(2)} .
\end{aligned}
$$

The term (2) above will not change if $X_{i}$ is replaced by $X_{i}^{*}$, neither will any product in (1). Then, given (B.9), we conclude that, if $i \notin \alpha, S_{\alpha}^{*} \geq S_{\alpha}$.

Proof of Proposition 4.9. Note that

$$
S_{T_{i}}=\left[1+\frac{\sum_{\alpha \not \supset i} \operatorname{Var}\left(f_{\alpha}\left(X_{\alpha}\right)\right.}{\operatorname{Var}\left(f_{T_{i}}(X)\right)}\right]^{-1} .
$$

Proposition 4.6 gives

$$
\begin{aligned}
\operatorname{Var}\left(f_{T_{i}}(X)\right)= & \operatorname{Var}\left(\varphi_{1}\left(X_{i}\right)\right) \prod_{j \neq i} \mathbb{E}\left(g_{j}\left(X_{j}\right)\right)^{2}+\operatorname{Var}\left(\varphi_{2}\left(X_{i}\right)\right) \\
& +\operatorname{Cov}\left(\varphi_{1}\left(X_{i}\right), \varphi_{2}\left(X_{i}\right)\right) \prod_{j \neq i} \mathbb{E}\left(g_{j}\left(X_{j}\right)\right)
\end{aligned}
$$

and if $i \notin \alpha$,

$$
\begin{gathered}
f_{\alpha}\left(X_{\alpha}\right)=\mathbb{E}\left(\varphi_{1}\left(X_{i}\right)\right) \prod_{j \notin \alpha} \mathbb{E}\left(g_{j}\left(X_{j}\right)\right) \times \prod_{j \in \alpha}\left(g_{j}\left(X_{j}\right)-\mathbb{E}\left(g_{j}\left(X_{j}\right)\right)\right), \\
\operatorname{Var}\left(f_{\alpha}\left(X_{\alpha}\right)\right)=\mathbb{E}\left(\varphi_{1}\left(X_{i}\right)\right)^{2} \prod_{j \notin \alpha} \mathbb{E}\left(g_{j}\left(X_{j}\right)\right)^{2} \times \operatorname{Var}\left(\prod_{j \in \alpha}\left(g_{j}\left(X_{j}\right)-\mathbb{E}\left(g_{j}\left(X_{j}\right)\right)\right) .\right.
\end{gathered}
$$


Then, $S_{T_{i}}^{*} \leq S_{T_{i}}$ holds if and only if

$$
\begin{gathered}
\frac{\operatorname{Var}\left(\varphi_{1}\left(X_{i}^{*}\right)\right) \prod_{j \neq i} \mathbb{E}\left(g_{j}\left(X_{j}\right)\right)^{2}+\operatorname{Var}\left(\varphi_{2}\left(X_{i}^{*}\right)\right)+\operatorname{Cov}\left(\varphi_{1}\left(X_{i}^{*}\right), \varphi_{2}\left(X_{i}^{*}\right)\right) \prod_{j \neq i} \mathbb{E}\left(g_{j}\left(X_{j}\right)\right)}{\mathbb{E}\left(\varphi_{1}\left(X_{i}^{*}\right)\right)^{2} \sum_{\alpha \not \supset} \prod_{j \notin \alpha} \mathbb{E}\left(g_{j}\left(X_{j}\right)\right)^{2} \times \operatorname{Var}\left(\prod_{j \in \alpha}\left(g_{j}\left(X_{j}\right)-\mathbb{E}\left(g_{j}\left(X_{j}\right)\right)\right)\right)} \\
\leq \frac{\operatorname{Var}\left(\varphi_{1}\left(X_{i}\right)\right) \prod_{j \neq i} \mathbb{E}\left(g_{j}\left(X_{j}\right)\right)^{2}+\operatorname{Var}\left(\varphi_{2}\left(X_{i}\right)\right)+\operatorname{Cov}\left(\varphi_{1}\left(X_{i}\right), \varphi_{2}\left(X_{i}\right)\right) \prod_{j \neq i} \mathbb{E}\left(g_{j}\left(X_{j}\right)\right)}{\mathbb{E}\left(\varphi_{1}\left(X_{i}^{*}\right)\right)^{2} \sum_{\alpha \not i} \prod_{j \notin \alpha} \mathbb{E}\left(g_{j}\left(X_{j}\right)\right)^{2} \times \operatorname{Var}\left(\prod_{j \in \alpha}\left(g_{j}\left(X_{j}\right)-\mathbb{E}\left(g_{j}\left(X_{j}\right)\right)\right)\right)} .
\end{gathered}
$$

With our hypothesis, we have that

$$
\begin{aligned}
& \frac{\operatorname{Var}\left(\varphi_{1}\left(X_{i}^{*}\right)\right)}{\mathbb{E}\left(\varphi_{1}\left(X_{i}^{*}\right)\right)^{2}} \leq \frac{\operatorname{Var}\left(\varphi_{1}\left(X_{i}\right)\right)}{\mathbb{E}\left(\varphi_{1}\left(X_{i}\right)\right)^{2}}, \\
& \frac{\operatorname{Var}\left(\varphi_{2}\left(X_{i}^{*}\right)\right)}{\mathbb{E}\left(\varphi_{1}\left(X_{i}^{*}\right)\right)^{2}} \leq \frac{\operatorname{Var}\left(\varphi_{2}\left(X_{i}\right)\right)}{\mathbb{E}\left(\varphi_{1}\left(X_{i}\right)\right)^{2}}
\end{aligned}
$$

and

$$
\frac{\operatorname{Cov}\left(\varphi_{1}\left(X_{i}^{*}\right), \varphi_{2}\left(X_{i}^{*}\right)\right)}{\mathbb{E}\left(\varphi_{1}\left(X_{i}^{*}\right)\right)^{2}} \leq \frac{\operatorname{Cov}\left(\varphi_{1}\left(X_{i}\right), \varphi_{2}\left(X_{i}\right)\right)}{\mathbb{E}\left(\varphi_{1}\left(X_{i}\right)\right)^{2}}
$$

This leads to the announced result.

Acknowledgements. We would like to thank the referees for their pertinent remarks, which have greatly improved the quality of the paper.

\section{REFERENCES}

[1] R.J. Beckman and M.D. McKay, Monte Carlo estimation under different distributions using the same simulation. Technometrics 29 (1987) 153-160.

[2] E. Borgonovo, Measuring uncertainty importance: investigation and comparison of alternative approaches. Risk Anal. 26 (2006) 1349-1361.

[3] E. Borgonovo, A new uncertainty importance measure. Reliab. Eng. Syst. Saf. 92 (2007) 771-784.

[4] E. Borgonovo and L. Peccati, On the Quantification and Decomposition of Uncertainty, Vol. 41. Springer (2007).

[5] M. Brigo and F. Mercurio, Interest Rate Models - Theory and Practice. Springer (2006).

[6] G.T. Buzzard and D. Xiu, Variance-based global sensitivity analysis via sparse-grid interpolation and cubature. Commun. Comput. Phys. 9 (2011) 542-567.

[7] P. Carr and D. Madan, Option valuation using the fast Fourier transform. J. Comput. Finance 2 (1999) 61-73.

[8] M. Denuit, J. Dhaene, M. Goovaerts and R. Kaas, Actuarial Theory for Dependent Risks: Measures, Orders and Models. Wiley (2005).

[9] E. Fagiuoli, F. Pellerey and M. Shaked, A characterization of the dilation order and its applications. Stat. Pap. 40 (1999) 393-406.

[10] S.L. Heston, A closed-form solution for options with stochastic volatility with applications to bond and currency options. Rev. Financ. Stud. 6 (1993) 327-343.

[11] B. Iooss, F. Van Dorpe and N. Devictor, Response surfaces and sensitivity analyses for an environmental model of dose calculations. Reliab. Eng. Syst. Saf. 91 (2006) 1241-1251.

[12] A. Janon, Analyse de sensibilité et réduction de dimension. Application à l'océanographie. Ph.D. thesis, Université de Grenoble (2012).

[13] S. Kochar, X. Li and M. Shaked, The total time on test transform and the excess wealth stochastic orders of distributions. Adv. Appl. Probab. 34 (2002) 826-845. 
[14] F.Y. Kuo, I.H. Sloan, G.W. Wasilkowski and H. Wozniakowski, On decompositions of multivariate functions. Math. Comput. 79 (2010) 953-966.

[15] P. Lemaître, E. Sergienko, A. Arnaud, N. Bousquet, F. Gamboa and B. Iooss, Density modification-based reliability sensitivity analysis. J. Stat. Comput. Simul. 85 (2015) 1200-1223.

[16] T.A. Mara and S. Tarantola, Variance-based sensitivity indices for models with dependent inputs. Reliab. Eng. Syst. Saf. 107 (2012) 115-121.

[17] A. Müller and D. Stoyan, Comparison Methods for Stochastic Models and Risks. Wiley (2002).

[18] A.B. Owen, Sobol'indices and shapley value. SIAM/ASA J. Uncertain. Quantif. 2 (2014) 245-251.

[19] F. Pappenberger, K. Beven, M. Ratto and P. Matgen, Multi-method global sensitivity analysis of flood inundation models. Adv. Water Resour. 31 (2008) 1-14.

[20] M. Rodriguez-Fernandez, J.R. Banga and F.J. Doyle, Novel global sensitivity analysis methodology accounting for the crucial role of the distribution of input parameters: application to systems biology models. Int. J. Robust Nonlinear Control 22 (2012) $1082-1102$.

[21] A. Saltelli, M. Ratto, T. Andres, F. Campolongo, J. Cariboni, D. Gatelli, M. Saisana and S. Tarantola, Global Sensitivity Analysis: The Primer. Wiley (2008).

[22] M. Shaked and J.G. Shanthikumar, Two variability orders. Probab. Eng. Inf. Sci. 12 (1998) 1-23.

[23] M. Shaked and J.G. Shanthikumar, Stochastic Orders. Springer (2007).

[24] M. Shaked, M.A. Sordo and A. Suarez-Llorens, A class of location-independent variability orders, with applications. J. Appl. Probab. 47 (2010) 407-425.

[25] I.M. Sobol, Global sensitivity indices for nonlinear mathematical models and their Monte Carlo estimates. Math. Comput. Simul. 55 (2001) 271-280.

[26] A.W. van der Vaart, Asymptotic Statistics. Vol. 3 of Cambridge Series in Statistical and Probabilistic Mathematics. Cambridge University Press, Cambridge (1998).

[27] H. Varella, M. Guérif and S. Buis, Global sensitivity analysis measures the quality of parameter estimation: the case of soil parameters and a crop model. Environ. Model. Softw. 25 (2010) 310-319.

[28] E. Volkova, B. Iooss and F. Van Dorpe, Global sensitivity analysis for a numerical model of radionuclide migration from the RRC "Kurchatov Institute" radwaste disposal site. Stoch. Environ. Res. Risk Assess. 22 (2008) 17-31. 